\title{
Differing effects of high-fat or high-carbohydrate meals on food hedonics in overweight and obese individuals
}

\author{
Mark Hopkins $^{1,2 *}$, Catherine Gibbons ${ }^{2}$, Phillipa Caudwell ${ }^{2,3}$, John E. Blundell ${ }^{2}$ and Graham Finlayson ${ }^{2}$ \\ ${ }^{1}$ Academy of Sport and Physical Activity, Faculty of Health and Wellbeing, Sheffield Hallam University, Sheffield S1O $2 B P$, UK \\ ${ }^{2}$ Institute of Psychological Sciences, Faculty of Medicine and Health, University of Leeds, Leeds LS2 9JT, UK \\ ${ }^{3}$ Medical and Healthcare Affairs, AstraZeneca, Horizon Place, 600 Capability Green, Luton LU1 3LU, UK
}

(Submitted 19 October 2015 - Final revision received 21 December 2015 - Accepted 10 February 2016 - First published online 22 March 2016)

\section{Abstract}

Although the effects of dietary fat and carbohydrate on satiety are well documented, little is known about the impact of these macronutrients on food hedonics. We examined the effects of ad libitum and isoenergetic meals varying in fat and carbohydrate on satiety, energy intake and food hedonics. In all, sixty-five overweight and obese individuals $\left(\mathrm{BMI}=30 \cdot 9(\mathrm{sD} 3 \cdot 8) \mathrm{kg} / \mathrm{m}^{2}\right)$ completed two separate test meal days in a randomised order in which they consumed high-fat/low-carbohydrate (HFLC) or low-fat/high-carbohydrate (LFHC) foods. Satiety was measured using subjective appetite ratings to calculate the satiety quotient. Satiation was assessed by intake at ad libitum meals. Hedonic measures of explicit liking (subjective ratings) and implicit wanting (speed of forced choice) for an array of HFLC and LFHC foods were also tested before and after isoenergetic HFLC and LFHC meals. The satiety quotient was greater after ad libitum and isoenergetic meals during the LFHC condition compared with the HFLC condition $(P=0.006$ and $P=0.001$, respectively), whereas ad libitum energy intake was lower in the LFHC condition $(P<0 \cdot 001)$. Importantly, the LFHC meal also reduced explicit liking $(P<0 \cdot 001)$ and implicit wanting $(P=0 \cdot 011)$ for HFLC foods compared with the isoenergetic HFLC meal, which failed to suppress the hedonic appeal of subsequent HFLC foods. Therefore, when coupled with increased satiety and lower energy intake, the greater suppression of hedonic appeal for high-fat food seen with LFHC foods provides a further mechanism for why these foods promote better short-term appetite control than HFLC foods.

\section{Key words: Explicit liking: Implicit wanting: Macronutrient composition: Energy intake: Satiety: Food hedonics}

The role of dietary carbohydrate in the aetiology and treatment of obesity is controversial, with some arguing that carbohydrate intake has a more prominent role in promoting overconsumption and weight gain than dietary fat ${ }^{(1,2)}$. Although this view has been strongly debated ${ }^{(3)}$, it has long been established that dietary macronutrients exert a hierarchical effect on appetite-related processes such as satiety and short-term food intake $^{(4)}$. When expressed relative to energy content rather than weight of food, protein exerts the strongest effect on satiety, followed by carbohydrate, whilst fat exerts the weakest effect ${ }^{(5)}$. This hierarchical effect has been demonstrated under a variety of laboratory and free-living conditions using subjective measures of appetite, biomarkers of satiety and food intake ${ }^{(6-11)}$. However, the underlying metabolic and, in particular, behavioural mechanisms that promote overconsumption following the consumption of energy-dense, high-fat foods are not well understood.

The differential effects of dietary macronutrients on satiety may relate to differences in pre-ingestive cognitive and sensory signals generated at the time of consumption ${ }^{(12)}$ and/or the post-ingestive metabolic effects of these foods ${ }^{(13-15)}$. However, recent evidence suggests that the hedonic value of foods encountered after consumption (e.g. food liking and wanting), which is closely linked to the perceived taste and energy content can also influence appetite and energy intake ${ }^{(16)}$. For example, a heightened liking (the perceived pleasurable sensory properties of food) and wanting (the attraction towards a specific food over available alternatives ${ }^{(17)}$ ) for high-fat, high-sweet foods has been noted in overweight and obese individuals $^{(18)}$ and those who demonstrate binge eating ${ }^{(19)}$. Despite this, the effect of macronutrient composition on food hedonics has received little attention, and existing data are contradictory.

While high-protein meals ( $25 \%$ of total energy) have been shown not to effect food hedonics compared with isoenergetic low-protein meals $(7 \% \text { of total energy) })^{(20)}$, Lemmens et al. ${ }^{(21)}$ reported that a meal containing $65 \%$ of its total energy from protein reduced 'wanting' to a greater extent than an isoenergetic high-carbohydrate meal (65\% of total energy). Furthermore, a 14-d low-protein diet $(0.5 \mathrm{~g}$ protein $/ \mathrm{kg}$ body

Abbreviations: HFLC, high-fat/low-carbohydrate foods; LFHC, low-fat/high-carbohydrate foods; LFPQ, Leeds Food Preference Questionnaire; SQ, satiety quotient.

* Corresponding author: Dr M. Hopkins, email M.Hopkins@shu.ac.uk 
Table 1. Descriptive characteristics for participants $(n 65)^{\star}$ (Mean values and standard deviations)

\begin{tabular}{|c|c|c|c|c|c|c|}
\hline & \multicolumn{2}{|c|}{ Whole group } & \multicolumn{2}{|c|}{ Males } & \multicolumn{2}{|c|}{ Females } \\
\hline & Mean & SD & Mean & SD & Mean & SD \\
\hline Age (years) & $41 \cdot 3$ & $8 \cdot 7$ & 41.5 & $7 \cdot 7$ & $41 \cdot 3$ & $9 \cdot 3$ \\
\hline BMI $\left(\mathrm{kg} / \mathrm{m}^{2}\right)$ & $30 \cdot 9$ & 3.8 & 30.6 & $4 \cdot 4$ & 31.0 & 3.5 \\
\hline Body fat (\%) & $39 \cdot 3$ & 7.5 & 32.8 & 5.9 & 43.6 & $5 \cdot 2$ \\
\hline Fat mass $(\mathrm{kg})$ & $35 \cdot 4$ & $9 \cdot 3$ & $32 \cdot 8$ & $10 \cdot 8$ & $37 \cdot 2$ & 7.9 \\
\hline Fat-free mass $(\mathrm{kg})$ & 54.5 & $10 \cdot 4$ & 64.8 & $6 \cdot 8$ & $47 \cdot 7$ & 5.9 \\
\hline RMR (kJ/d) & $7349 \cdot 2$ & $1425 \cdot 5$ & $8522 \cdot 8$ & $1185 \cdot 7$ & $6519 \cdot 9$ & 828.0 \\
\hline
\end{tabular}

* Body composition was measured using air displacement plethysmography, whereas RMR was measured using indirect calorimetry. Details of the procedures used can be found elsewhere ${ }^{(26)}$

weight-per d) was found to increase protein intake, wanting, preference for savoury high-protein foods ${ }^{(22)}$ and the neural activation to savoury food cues in brain reward regions ${ }^{(23)}$ compared with a high-protein diet $(2.0 \mathrm{~g}$ protein $/ \mathrm{kg}$ body weight per·d).

Although these data suggest that dietary macronutrients may also differentially affect food hedonics, the acute effects of macronutrient composition, and in particular dietary fat, on food hedonics has yet to be examined. Given the controversy over the relative contribution of dietary fat and carbohydrate in promoting overconsumption and weight gain, this warrants further attention. Therefore, the aim of this study was to examine the effects of ad libitum and isoenergetic meals varying in dietary fat and carbohydrate on energy intake, satiety and food hedonics in overweight and obese individuals.

\section{Methods \\ Participants}

In all, sixty-five overweight and obese males ( $n$ 26) and females ( $n$ 39) were recruited onto this randomised, cross-over design study. Descriptive characteristics of participants are displayed in Table 1. All participants were non-smokers, physically inactive ( $\leq 2 \mathrm{~h}$ /week of exercise over the previous 6 months), weight stable ( $\pm 2 \mathrm{~kg}$ for the previous 3 months) and not taking medication known to affect metabolism or appetite. This study was conducted according to the guidelines laid down in the Declaration of Helsinki, and ethical approval was granted by the Leeds West National Health Service (NHS) Research Ethics Committee (09/H1307/7). All participants provided written informed consent before taking part. The project was registered under international standard identification for controlled trials ISRCTN47291569.

\section{Study design}

Participants completed two separate probe test meal days in a randomised order in which they consumed either high-fat/ low-carbohydrate (HFLC) or low-fat/high-carbohydrate (LFHC) meals across the day that were matched for sensory properties and taste. Total daily energy intake was measured using a laboratory-based test meal design that included fixed-energy and ad libitum meals, whereas satiation (energy intake during a single meal) was measured during ad libitum meal consumption only. Satiety was measured using subjective appetite ratings adjusted for energy intake from the breakfast and lunch meals to calculate the satiety quotient $(\mathrm{SQ})^{(24)}$. Hedonic measures of explicit liking (subjective ratings) and implicit wanting (speed of forced choice) for an array of HFLC and LFHC foods were also tested before and after the isoenergetic lunch meal using the Leeds Food Preference Questionnaire (LFPQ) ${ }^{(25)}$.

\section{Procedures}

Total daily energy intake and satiation. Total daily energy intake and satiation (measured via energy intake during a single meal) were measured using a laboratory-based test meal protocol in which participants consumed either HFLC or LFHC foods across the whole day. Test days were separated by at least $2 \mathrm{~d}$, and the order in which participants performed the HFLC and LFHC days was randomised and counter-balanced. The form of the meals on each test day was identical, with foods similar in appearance and taste acceptability so that participants could not detect the nutritional manipulation. The mean proportion of energy contributed by fat, protein and carbohydrate to total daily energy intake on the HFLC and LFHC test days was 56.0 (sD 3.2), 13.9 (sD 2.1) and 30.1 (sD 3.9)\%, and 23.0 (SD 3.3), 13.5 (sD 1.5) and 63.5 (SD 4.4)\%, respectively. Mean taste acceptability for the HFLC and LFHC conditions was assessed using visual analogue scales in a sub-sample of participants ( $n$ 16) who took part in the wider study, and no differences existed between the HFLC $(62.3(\mathrm{SD} 7.2) \mathrm{mm})$ and LFHC conditions $(56.9$ (sD 6.1) $\mathrm{mm} ; P=0 \cdot 242)$. Similarly, mean food satisfaction was also assessed using visual analogue scales following the HFLC (63.8 (SD 7.9) mm) and LFHC (62.2 (sD 6.6$) \mathrm{mm}$ ) conditions, and again, no differences existed between conditions $(P=0 \cdot 724)$.

During the test days, participants consumed an ad libitum breakfast meal, a fixed-energy lunch (3347 kJ (800 kcal)) and an ad libitum dinner meal ( $4 \mathrm{~h}$ apart). After the dinner meal, participants were free to leave the research laboratory but were given an ad libitum snack box of foods to consume if desired during the evening. All meals provided on the test day were either HFLC or LFHC, and participants were required to consume only the foods and drinks provided on these test days. Details of the individual food items, macronutrient composition 
and weight of food consumed can be found in the online Supplementary Table S1 and elsewhere ${ }^{(26)}$.

All meals consumed in the research unit were eaten in isolation, with participants instructed to eat as much or as little as they wanted until they were comfortably full during ad libitum meal consumption. Food was provided in excess of expected consumption, with participants able to request further food or water if required. Before participation, individuals completed a food preference questionnaire, and if they strongly disliked any of the test foods, participants were excluded if a suitable alternative (matched for macronutrient composition) could not be found. Energy intake was calculated by weighing the food before and after consumption (to the nearest $0.1 \mathrm{~g}$ ), and with reference to the manufacturers' energy values. To calculate test meal energy intake, the energy equivalences used for protein, fat and carbohydrate were 17,38 and $16 \mathrm{~kJ} / \mathrm{g}$ (4, 9 and $3.75 \mathrm{kcal} / \mathrm{g}$ ), respectively. Total daily energy intake was taken as the energy consumed during the breakfast, lunch and dinner meals, and intake from the snack box. Energy intake during the ad libitum breakfast and dinner meals was used to represent satiation in the present paper.

Subjective appetite ratings. Subjective ratings of appetite were measured during test meal probe days using visual analogue scales presented on a validated hand-held electronic appetite rating system (EARS II) ${ }^{(27)}$. On each day, ratings were recorded immediately before and after a meal, and at hourly intervals throughout the day (from 08.00 to 18.00 hours). The use of visual analogue scales for the measurement of subjective appetite has previously been shown to be valid and reproducible $^{(28)}$. Furthermore, visual analogue scales have been used to detect changes in appetite following manipulations of energy intake ${ }^{(29,30)}$ and diet composition ${ }^{(31)}$, whereas the EARS II electronic rating system has been validated against the traditional pen and paper technique ${ }^{(27)}$.

Satiety. The suppression of hunger per calorie of intake for the ad libitum breakfast meal and fixed-energy lunch meals was assessed by calculating the SQ. The SQ was developed by Green et $a l^{(24)}$, and it expresses changes in postprandial appetite ratings relative to the energy content of a meal. As such, it reflects the capacity of a meal to modulate the strength of postprandial satiety sensations. The SQ of a meal was calculated using the following formula using subjective hunger ratings ${ }^{(24)}$, with a higher SQ indicative of a greater satiating efficiency:

\section{Satiety quotient}

$$
=\frac{\text { rating pre-eating episode }- \text { rating post-eating episode }}{\text { intake of eating episode }} \times 100 \text {. }
$$

It has been suggested that the SQ provides a better marker of satiety than postprandial hunger ratings, as it takes into account both the pre-meal appetite sensations and the energy content of the meal consumed ${ }^{(32)}$. The SQ has also been shown to be associated with ad libitum food intake following a variety of nutritional interventions ${ }^{(32,33)}$
Hedonic assessment of high-fat/low-carbohydrate and low-fat/high-carbohydrate foods. Immediately before and after the fixed-energy lunch meal, the hedonic profile of an array of foods was assessed using the LFPQ ${ }^{(25)}$. The LFPQ provides measures of different components of food preference and hedonics. Participants are presented with an array of pictures of individual food items common in the diet. Foods in the array are chosen by the experimenter from a validated database to be either predominantly high ( $>45 \%$ energy) or low ( $<20 \%$ energy) in fat but similar in familiarity, protein content, sweet or nonsweet taste and acceptability. Each food category was represented by eight photographs of ready-to-eat foods. Details of the mean energy density, serving and macronutrient composition of food items and categories' used in the LFPQ can be found in Table 2. The LFPQ has been validated against physiological and behavioural end points in a range of research ${ }^{(34-36)}$. The specific end points examined from the LFPQ were explicit liking, implicit wanting and food preference for HFLC relative to LFHC foods, as described below. The LFPQ has been shown to demonstrate reliable immediate and post-meal changes ${ }^{(37)}$, and it is a good predictor of food choice and intake in laboratory and community-based samples ${ }^{(22,38)}$.

Explicit liking and implicit wanting. To measure explicit liking, participants rated the extent to which they liked each food (e.g. how pleasant would it be to taste this food now?). The food images were presented individually in a randomised order and participants made their ratings using a 100-mm visual analogue scale.

Implicit wanting was assessed using a forced choice methodology in which the food images were paired so that every image from each food category was compared with every other type over ninety-six trials (food pairs). Participants were instructed to respond as quickly and accurately as they could, indicating the food they want to eat the most at that time (e.g. which food do you most want to eat now?). Following Dalton et al. ${ }^{(39)}$, the food pair trials were presented in three blocks, with each stimulus appearing eight times. Stimuli were presented until a valid response was detected up to a maximum of $4000 \mathrm{~ms}$ with a variable 500-1000 ms washout between presentations in which a central fixation cross was displayed. To measure implicit wanting, reaction times for all responses were covertly recorded and used to compute mean response times for each food type after adjusting for frequency of selection. Therefore, a positive score indicates a more rapid preference for high-fat foods over low-fat foods and a negative score indicates the opposite. A score of 0 indicates that high-fat and low-fat foods are equally preferred. A frequency-weighted algorithm was used so the implicit wanting score could be influenced by both selection (positively contributing to the score) and non-selection (negatively contributing to the score) of food type.

\section{Statistical analysis}

Data are reported as means with their standard errors throughout unless otherwise stated. Statistical analyses were performed using IBM SPSS for Windows (version 21). Where appropriate, Greenhouse-Geisser probability levels were used 


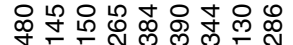

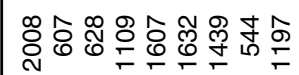

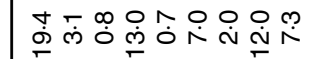

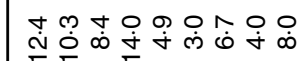

$\circ$

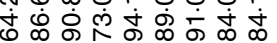

昜

莺

$8)$

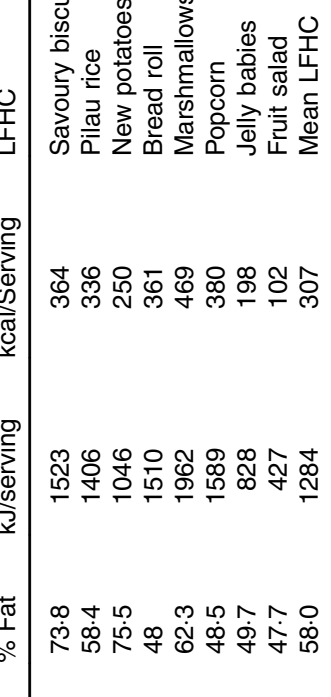

동

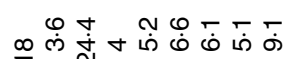

$\circ$

옹

$\circ$

(

造

号

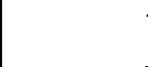

志

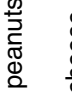

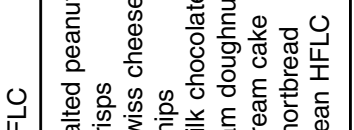

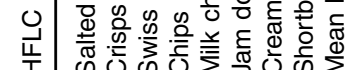

to adjust for sphericity, and Bonferroni adjustments were applied to control for multiple post hoc comparisons. Our sample size of sixty-five was assessed for adequate power by a posteriori power analysis using $\mathrm{G}^{*}$ Power $^{(40)}$ to find an effect of macronutrient composition on implicit wanting for HFLC food, based on data from Griffioen-Roose et al. ${ }^{(35)}$, and expected correlation of $0.5, \beta=0.8$ and $\alpha=0.05$. A paired $t$ test was used to examine differences between pre-meal subjective appetite ratings (hunger and fullness) and total daily energy intake during the HFLC and LFHC conditions. The effects of macronutrient composition on satiation (i.e. energy intake during breakfast and lunch meals) were examined using a two-way ANOVA (meal $\times$ macronutrient composition) with repeated measures. Similarly, the effect of macronutrient composition on satiety (SQ) was examined following the ad libitum breakfast and fixed-energy lunch meals using separate two-way ANOVAs (time $\times$ macronutrient composition) with repeated measures.

For LFPQ measures, mean scores for HFLC and LFHC categories were computed for implicit wanting and explicit liking outcomes. Mean LFHC scores were then subtracted from the mean for HFLC scores to provide a composite score representing hedonic value for HFLC relative to LFHC food for liking and wanting. Using this approach, a positive score indicated greater liking or wanting for HFLC foods over LFHC foods; a negative score indicated greater liking or wanting for LFHC foods over HFLC foods, and a score of 0 indicated an equal liking or wanting for HFLC and LFHC foods. The explicit liking and implicit wanting appeal bias scores were examined separately using a two-way ANOVA (macronutrient composition $\times$ hunger state) with repeated measures. Interactions were explored further using simple post hoc comparisons. To test whether hedonic end points were associated with food intake, simple linear regression was used to examine the relationships between explicit liking and implicit wanting and ad libitum dinner meal intake.

\section{Results}

The effect of macronutrient composition on appetite, satiation and total daily energy intake

No differences existed between the pre-breakfast ratings of subjective hunger (63.3 (sем 2.9) $v .60 .8$ (S巨м 3.1) $\mathrm{mm} ; P=0.509)$ and fullness (19.9 (SEM 2.34) v. 24.4 (SEM 2.8) $\mathrm{mm} ; P=0.138$ ) during HFLC and LFHC conditions, respectively. Similarly, no differences existed in ratings of hunger (62.3 (SEM 3.0) v. 63.7 (SEM 3.0$) \mathrm{mm} ; P=0.592$ ) and fullness (30.1 (SEM 2.6) $v .27 \cdot 3$ (SEM 2.6$) \mathrm{mm} ; P=0.320$ ) immediately before the lunch meal during HFLC and LFHC conditions, respectively.

Total daily energy intake was significantly greater during the HFLC condition compared with the LFHC condition (4143.8 (sem 338.9) kJ (990.4 (SEM 81.0) kcal); $P<0.001$ ). As expected, no differences existed in energy intake during the fixed-energy HFLC $(3346.8$ (SEM 9.6) kJ (799.9 (SEM 2.3) kcal)) and LFHC (3287.8 (S巨M 12.1) kJ (785.8 (s巨M 2.9) kcal); $P>0.05$ ) lunch meals. To examine the effects of macronutrient composition on satiation (i.e. energy intake during a single meal) during the ad libitum breakfast and dinner meals, energy intake during the separate test 
meals was examined. A two-way ANOVA (meal× macronutrient composition) with repeated measures indicated a significant main effect of meal $\left(F_{2.54,162.81}=35.926 ; P<0.001 ; \eta^{2}=0.360\right)$ and macronutrient composition $\left(F_{1,64}=156.953 ; P<0.001 ; \eta^{2}=0.710\right)$. There was also a significant meal $\times$ macronutrient composition interaction $\left(F_{2 \cdot 10,134 \cdot 64}=36 \cdot 045 ; P<0.001 ; \eta^{2}=0.360\right)$, such that energy intake was significantly higher at breakfast $(1411.8$ (SEM 184.9) kJ (337.2 (SEM 44.2) kcal); $P<0.001)$ and dinner (2225.0 (SEM 147.2) kJ (531.8 (SEM 35.2) kcal); $P<0.001)$ during the HFLC condition compared with the LFHC condition (Fig. 1).

\section{The effect of macronutrient composition on satiety following ad libitum breakfast meal consumption}

There was a significant effect of macronutrient composition on SQ following the consumption of the ad libitum breakfast meal,

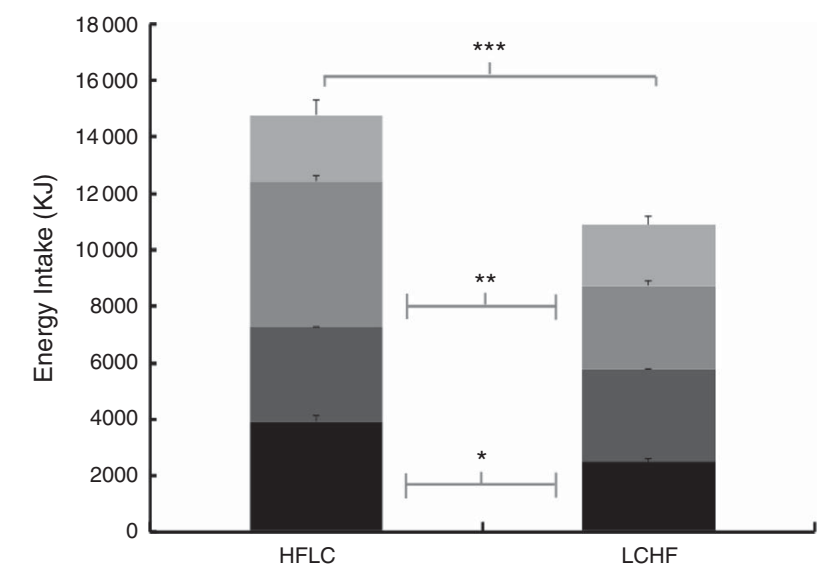

Fig. 1. Mean total daily energy intake and energy intake during separate meals during the high-fat/low-carbohydrate (HFLC) and low-fat/high-carbohydrate (LFHC) conditions. Values are means, with their standard errors represented by vertical bars. ${ }^{\star}$ Significant difference in breakfast intakes $(P<0.05)$. ${ }^{\star}$ Significant difference in dinner intakes $(P<0 \cdot 05)$. ${ }^{\star * *}$ Significant difference in total daily energy intake as indicated by a two-way ANOVA with repeated measures $(P<0.05)$. , Snack box intake; , dinner intake; , lunch intake; $\square$, breakfast intake.

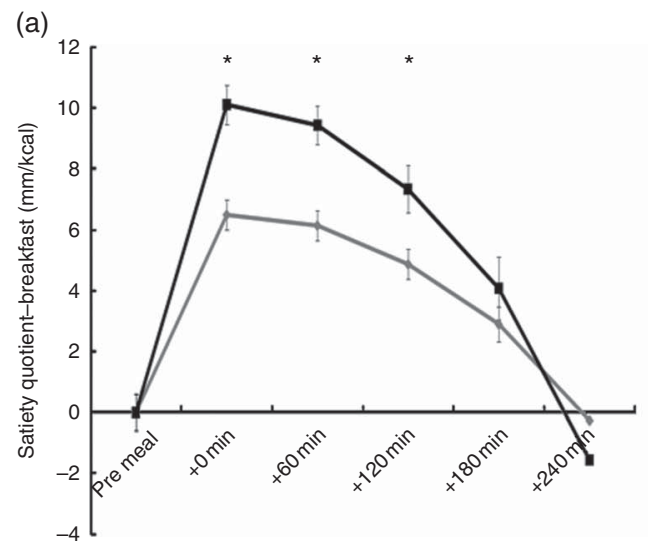

with a two-way ANOVA (time $\times$ macronutrient composition) with repeated measures indicating a significant main effect of time $\left(F_{1.49,95.49}=97.024 ; P<0.001 ; \eta^{2}=0.603\right)$ and macronutrient composition $\left(F_{1,64}=8.072 ; \quad P=0.006 ; \quad \eta^{2}=0 \cdot 112\right)$. Furthermore, there was a significant time $\times$ macronutrient composition interaction $\quad\left(F_{2 \cdot 27,143.20}=19 \cdot 687 ; \quad P<0 \cdot 001\right.$; $\eta^{2}=0 \cdot 235$ ), such that the LFHC breakfast SQ was significantly higher than the HFLC breakfast SQ immediately after $(P<0.001)$ and at $60(P<0.001)$ and $120 \mathrm{~min}$ post-meal consumption ( $P=0 \cdot 001$; Fig. 2$)$.

\section{The effect of macronutrient composition on satiety following consumption of the isoenergetic lunch meal}

There was also an effect of macronutrient composition on SQ following consumption of the fixed-energy lunch meal (Fig. 2), with a two-way ANOVA (time $\times$ macronutrient composition) with repeated measures indicating a significant main effect of time $\left(F_{2 \cdot 56,164 \cdot 38}=109 \cdot 980 ; P<0 \cdot 001 ; \eta^{2}=0.632\right)$. There was also a significant main effect of macronutrient composition $\left(F_{1,64}=11 \cdot 314 ; P=0 \cdot 001 ; \eta^{2}=0 \cdot 150\right)$, such that SQ was significantly higher following consumption of the LFHC meal compared with the HFLC meal $(P=0 \cdot 001)$. However, there was no time $\times$ macronutrient composition interaction $\left(F_{2 \cdot 96,189 \cdot 57}=0 \cdot 187 ; P=0 \cdot 945 ; \eta^{2}=0 \cdot 003\right)$.

\section{The effect of macronutrient composition on the hedonic assessment of food following isoenergetic meal consumption}

When the explicit liking score for HFLC relative to LFHC foods was examined, a two-way ANOVA with repeated measures indicated a significant main effect of macronutrient composition $\left(F_{1,64}=8.432 ; P=0.005 ; \eta^{2}=0 \cdot 116\right)$, such that explicit liking for HFLC foods was greater during the HFLC condition. There was also a significant macronutrient composition $\times$ hunger state interaction $\left(F_{1,64}=5.993 ; \quad P=0.017 ; \quad \eta^{2}=0.086\right)$. Although explicit liking did not differ between conditions in the hungry state, that is, before meal $(P=0.519)$, explicit liking for HFLC (b)

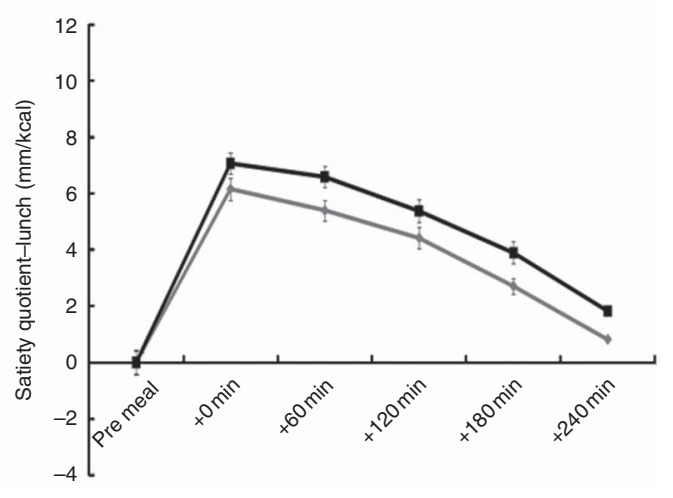

Fig. 2. Mean postprandial changes in the satiety quotient following the consumption of ad libitum high-fat/low-carbohydrate (HFLC, $\longrightarrow$ ) and low-fat/highcarbohydrate (LFHC, - breakfast (a) and fixed-energy lunch meals (b). Values are means, with their standard errors represented by vertical bars. ${ }^{*}$ Significant difference in the satiety quotient between conditions as indicated by a two-way ANOVA with repeated measures $(P<0.01)$. 
(a)

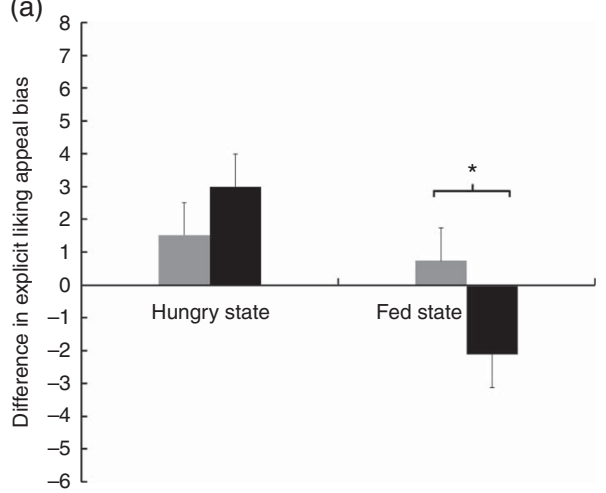

(b)

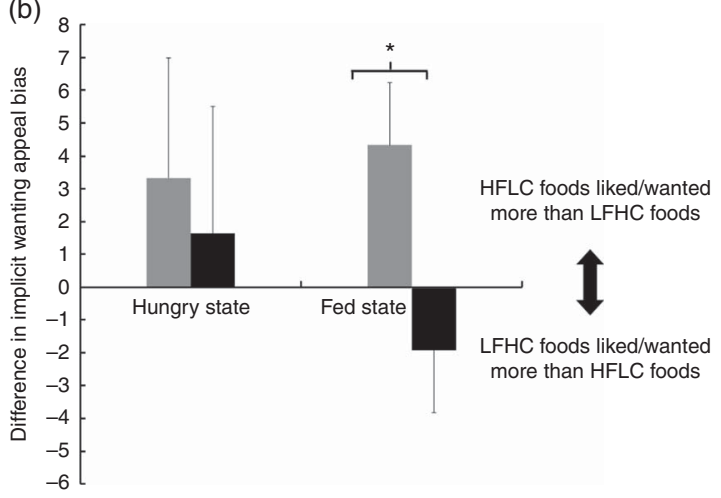

Fig. 3. Mean explicit liking (a) and implicit wanting (b) appeal bias scores for high-fat foods relative to low-fat foods before and after consumption of isoenergetic high-fat/low-carbohydrate (HFLC, $\square$ ) and low-fat/high-carbohydrate (LFHC, $\square$ ) meals. Values are means, with their standard errors represented by vertical bars. *Significant difference in energy intake between conditions as indicated by a two-way ANOVA with repeated measures $(P<0.05)$.

Table 3. Measures of explicit liking and implicit wanting and ad libitum dinner intake during the high-fat/low-carbohydrate (HFLC) and low-fat/highcarbohydrate (LFHC) conditions ( $n$ 65)†

(Correlation coefficients)

\begin{tabular}{lcccc}
\hline & Explicit liking: hungry state & Explicit liking: fed state & Implicit wanting: hungry state & Implicit wanting: fed state \\
\hline HFLC dinner intake (kcal) & $r 0.313^{\star}$ & $r 0.302^{\star}$ & $r 0.271^{\star}$ & $r 0.408^{\star \star}$ \\
& $R^{2} 0.098$ & $R^{2} 0.091$ & $R^{2} 0.074$ & $R^{2} 0.167$ \\
LFHC dinner intake (kcal) & $r 0.342^{\star}$ & $r 0.369^{\star}$ & $r 0.315^{\star}$ & $r 0.453^{\star \star}$ \\
& $R^{2} 0.117$ & $R^{2} 0.136$ & $R^{2} 0.099$ & $R^{2} 0.206$ \\
\hline
\end{tabular}

${ }^{\star} P<0.05,{ }^{* \star} P<0.001$.

† Simple linear regression was used to examine the relationships between explicit liking and implicit wanting and ad libitum dinner meal intake.

foods was significantly lower in the fed state following the consumption of the LFHC meal compared with the HFLC meal $(P<0 \cdot 001$; Fig. 3).

When the implicit wanting score for HFLC relative to LFHC foods was examined, a significant main effect of macronutrient composition was seen $\left(F_{1,64}=4 \cdot 846 ; P=0.031\right.$; $\left.\eta^{2}=0.070\right)$, such that implicit wanting was higher during the HFLC condition. There was no main effect of hunger state $\left(F_{1,64}=0.205 ; P=0.652 ; \eta^{2}=0.001\right)$, and the macronutrient composition $\times$ hunger state interaction approached significance $\left(F_{1,64}=2.851 ; \quad P=0.096 ; \quad \eta^{2}=0.043\right)$. As can be seen in Fig. 3, consumption of the HFLC meal increased wanting (1.00 (SEM 2.92)), whereas LFHC foods decreased wanting (-3.57 (sem 3.35)). Post boc comparisons indicated that implicit wanting for HFLC foods did not differ between conditions in the hungry state (i.e. before meal; $P=0.427$ ), but it was significantly lower in the fed state following the consumption of the LFHC meal compared with the HFLC meal $(P=0 \cdot 011$; Fig. 3).

\section{Association between the hedonic assessment of high-fat/} low-carbohydrate and low-fat/high-carbohydrate food and ad libitum food intake

To examine whether the hedonic assessment of food was associated with food intake, simple linear regression was used to examine the relationships between explicit liking, implicit wanting and ad libitum dinner intake. As can be seen in Table 3, positive associations were seen between explicit liking and implicit wanting (in the hungry and fed states) and ad libitum dinner intake during the HFLC and LFHC conditions.

\section{Discussion}

The aim of the present study was to examine the effects of macronutrient composition on energy intake, satiety and the post-ingestive hedonic assessment of subsequent foods. When participants were allowed to eat ad libitum, consumption of LFHC foods resulted in greater postprandial satiety (higher SQ values), greater satiation (lower self-selected meal intake) and lower total daily energy intake compared with the consumption of HFLC foods. Importantly, despite controlling for energy content, weight and palatability, the explicit liking and implicit wanting for high-fat foods were also suppressed to a greater extent following consumption of the LFHC lunch meal compared with the HFLC meal. As such, these data indicate that changing the composition of meals from HFLC to LFHC not only reduces energy intake and increases satiety, but also reduces the relative hedonic value of other HFLC food options. Taken together, these findings suggest that LFHC foods may promote better short-term appetite control than HFLC foods via both hedonic and appetite-based mechanisms. 
The effects of macronutrient composition on satiety and food intake

A clear effect of macronutrient composition on energy intake was observed in the present study, with total daily energy intake and self-selected intake (satiation) during the ad libitum breakfast and dinner meals significantly lower during the LFHC condition compared with the HFLC condition. There was also a strong effect of macronutrient composition on satiety, with the ad libitum LFHC breakfast found to be more satiating than the equivalent HFLC breakfast (as indicated by higher postprandial SQ scores). Indeed, the consumption of the LFHC breakfast increased satiety despite the lower energy content of the LFHC breakfast meal (and no differences in fasting hunger or fullness between conditions). This effect was transient, however, with no differences in SQ noted between conditions $180 \mathrm{~min}$ post consumption. The effect of macronutrient composition was also apparent under isoenergetic feeding conditions (albeit to a lesser extent), with greater SQ again seen after the LFHC lunch meal. In line with previous findings ${ }^{(5,8)}$, these data indicate that switching from HFLC to LFHC foods not only reduces energy intake but also increases the potency of postprandial satiety under ad libitum and isoenergetic feeding conditions.

Alterations in the physiological signals arising from the fat and carbohydrate content of the meals may underlie the differences in satiety seen in the present study, with the macronutrient composition of meals mediating the secretion of postprandial satiety hormones such as glucagon-like peptide-1 and peptide $\mathrm{YY}^{(13-15)}$. Mixed macronutrient meals that are representative of the natural local eating habits of the participants were used in the present study. The mean carbohydrate content during the HFLC was 30.1 (SD 3.9)\% (as opposed to 63.5 (SD 4.4$) \%$ in the LFHC condition), similar to that recommended by the recent Scientific Advisory Committee on Nutrition recommendations on carbohydrates ${ }^{(41)}$. As such, the carbohydrate content of the HFLC meals would have still stimulated the release/suppression of postprandial satiety hormones, but to a lesser extent than the LFHC meal. This may help account for why the differences in SQ between conditions were smaller under isoenergetic feeding conditions, a finding that has been previously reported ${ }^{(42)}$.

\section{The effect of macronutrient composition on food hedonics}

Although differences in the hedonic assessment of food is increasingly being recognised as a risk factor for overconsumption and weight gain ${ }^{(25)}$, the effect of macronutrient composition on the liking and wanting for subsequent foods has received little attention. Importantly, the present study demonstrated that explicit liking for high-fat foods was reduced to a greater extent following consumption of a LFHC test meal compared with a HFLC meal (despite controlling for the energy, weight and palatability of food). Furthermore, similar trend effects were observed for implicit wanting, with the LFHC meal decreasing wanting for high-fat foods, whereas the HFLC meal increased wanting for high-fat foods. These findings are interesting given that pre-meal appetite sensations (hunger and fullness) did not differ between conditions. It is also interesting to note that, when hungry, individuals preferred HFLC foods relative to LFHC foods to a similar degree during both conditions. This preference changed away from HFLC foods in the fed state during the LFHC condition, but remained during the HFLC condition. While this apparent dissociation during the fed state might counter-intuitively suggest that individuals increased their preference for the more satiating LFHC foods in the fed state during the LFHC condition (despite already being more satiated), the decreased appeal bias scores in the fed state during the LFHC condition are more likely to reflect a reduced preference for HFLC, rather than an increased preference for LFHC foods per se. Indeed, previous studies have shown that, when satiated, individuals tend to experience a reduced preference for HFLC compared with LFHC under ad libitum feeding conditions ${ }^{(25,37)}$. As such, it was interesting to observe in the present study that the consumption of HFLC food did not reduce liking or wanting for HFLC foods to the same extent as consumption of LFHC food under isoenergetic conditions. Therefore, a sustained liking and wanting for high-energy foods when satiated may throw new light on how high-fat diets lead to overconsumption.

The underlying mechanisms behind this macronutrient derived effect on food hedonics are unknown, but this may again be linked to the metabolic consequences of food ingestion. Leptin and insulin, which are both thought to tonically inhibit brain reward pathways ${ }^{(43)}$, are known to exhibit differential responses to dietary fat and carbohydrate ingestion $^{(44-46)}$. Although pre-breakfast ratings of hunger and fullness did not differ between conditions (indicating that participants started each condition with the same motivation to eat), it is possible that the response to breakfast may have also influenced the subsequent responses to lunch. However, no differences existed between conditions for pre-lunch subjective hunger, fullness or SQ, suggesting that the observed differences in post-meal liking and wanting were because of differences in the meal characteristics rather than a 'carryover' effect from breakfast.

These novel findings, found using a robust sample size ( $n$ 65) and a validated measure of food liking and wanting ${ }^{(34-36)}$, suggest a role for macronutrient composition in mediating the perceived hedonic value of food during the fed state. This is of importance, as the attenuated post-meal suppression of food liking and wanting following HFLC food consumption may pose as a risk factor for later snacking or larger subsequent meal intake. Indeed, in the present study, explicit liking and implicit wanting were positively associated with energy intake during the ad libitum dinner meal, indicating that the changes in food hedonics were expressed behaviourally through subsequent food intake (although differences in breakfast intake and satiety would have also influenced dinner intake). It is interesting to note that Lemmens et al. ${ }^{(21)}$ reported that the consumption of a high-protein, but not carbohydrate, meal reduced wanting. These data are in contrast to the current findings in which the LFHC meal actually suppressed liking and wanting for high-fat foods. However, although hedonic reward was measured using behaviourally relevant tasks during the present study, Lemmens et $a l .{ }^{(21)}$ measured wanting via engagement with memory games in a small sample of individuals ( $n$ 16) characterised by 
disinhibited eating behaviour (defined as a score $>5$ on the Three Factor Eating Questionnaire $\left.{ }^{(47)}\right)$.

It should be noted that while the present study indicates that LFHC foods dampen the hedonic appeal of high-fat foods to a greater extent than HFLC foods (while also resulting in greater satiety and lower energy intake), eating behaviour was only measured across a single day. As such, inferences about the long-term effects of a habitual LFHC diet on food hedonics cannot be made in the present study. However, Martin et al. ${ }^{(48)}$ has reported that individuals ( $n$ 134) following a 2-year low-carbohydrate diet were 'less bothered by hunger' and demonstrated decreased cravings for carbohydrates and preferences for high-carbohydrate and high-sugar foods compared with those following a low-fat diet ( $n$ 136). Furthermore, protein status after a 14-d high-protein diet has been shown to affect subsequent protein intake, wanting and preference for savoury, high-protein foods ${ }^{(22)}$ and neural activation in brain reward regions in response to savoury food cues $^{(23)}$. However, further research is needed to examine the long-term effects of diets varying in macronutrient composition on food hedonics.

The need for long-term studies examining the effects of macronutrient composition on food hedonics is emphasised by the ongoing debate regarding the effectiveness of diets differing in macronutrient composition on weight loss ${ }^{(49)}$. The present findings suggest that LFHC foods promote reduced energy intake, and they are in line with previous studies demonstrating that low-fat diets are effective for long-term weight loss ${ }^{(50)}$. However, inferences made about changes in body composition from studies that manipulate dietary intake acutely should be made cautiously. Indeed, recent findings have questioned whether low-fat diets are more effective than other isoenergetic dietary interventions for weight loss (i.e. low-carbohydrate or high-protein diets) ${ }^{(51)}$. It should also be noted that no control was made for menstrual cycle phase in female participants. This may have contributed to the variability seen in food hedonics, as studies have previously shown that eating behaviour and food hedonics are influenced to a small extent by the phases of the menstrual cycle ${ }^{(52,53)}$. Furthermore, this study only included overweight and obese individuals, and therefore no inferences can be made as to whether macronutrient composition also mediates food hedonics in lean individuals

\section{Conclusions}

When consumed under ad libitum and isoenergetic feeding conditions, HFLC foods have a weaker action on satiety and promote greater energy intake compared with LFHC foods. Importantly, HFLC foods also failed to dampen the subsequent appeal bias for high-fat foods compared with energy-, weightand palatability-matched LFHC foods. Therefore, these data demonstrate the acute impact of dietary fat and carbohydrate in moderating energy intake, and suggest that HFLC foods not only promote subsequent energy intake via effects on satiation and satiety but also through an effect on the subsequent hedonic value of food. Taken together, these data suggest that LFHC foods may help promote better short-term appetite control than HFLC foods.

\section{Acknowledgements}

Research relating to this study was funded by BBSRC (DRINC) grant no. BB/G005524/1 and European Union Seventh Framework Programme (FP7/2007-2013) under grant agreement no. 266408 'Full4Health'. Neither funding body had a role in the design, analysis or writing of this article. The project was registered under international standard identification for controlled trials ISRCTN47291569.

The authors' contributions are as follows: M. H., C. G. and P. C. contributed to the data collection and wrote the manuscript. C. G., J. E. B., G. F. and P. C. contributed to the study design. J. E. B. and G. F. contributed to the interpretation of data and writing of the manuscript. M. H. and G. F. contributed to the data analyses. All authors read and approved the final version of the manuscript.

The authors declare that there are no conflicts of interest.

\section{Supplementary material}

For supplementary material/s referred to in this article, please visit http://dx.doi.org/10.1017/S0007114516000775

\section{References}

1. Taubes G (2007) Good Calories, Bad Calories: Challenging the Conventional Wisdom on Diet, Weight Control, and Disease. New York: Alfred A. Knopf.

2. Taubes G (2011) Why We Get Fat: And What to Do About It. New York: Random House, Inc.

3. Hall KD, Bemis T, Brychta R, et al. (2015) Calorie for calorie, dietary fat restriction results in more body fat loss than carbohydrate restriction in people with obesity. Cell Metab 22, 427-436.

4. Blundell J, Lawton C, Cotton J, et al. (1996) Control of human appetite: implications for the intake of dietary fat. Annu Rev Nutr 16, 285-319.

5. Blundell JE, Burley V, Cotton J, et al. (1993) Dietary fat and the control of energy intake: evaluating the effects of fat on meal size and postmeal satiety. Am J Clin Nutr 57, 772S-777S.

6. Cotton JR, Burley VJ, Weststrate JA, et al. (1994) Dietary fat and appetite: similarities and differences in the satiating effect of meals supplemented with either fat or carbohydrate. J Hum Nutr Diet 7, 11-24.

7. Robinson TM, Gray RW, Yeomans MR, et al. (2005) Test-meal palatability alters the effects of intragastric fat but not carbohydrate preloads on intake and rated appetite in healthy volunteers. Physiol Behav 84, 193-203.

8. Lawton C, Burley V, Wales J, et al. (1993) Dietary fat and appetite control in obese subjects: weak effects on satiation and satiety. Int J Obes 17, 409-416.

9. Westerterp-Plantenga M, Rolland V, Wilson S, et al. (1999) Satiety related to $24 \mathrm{~h}$ diet-induced thermogenesis during high protein/carbohydrate vs high fat diets measured in a respiration chamber. Eur J Clin Nutr 53, 495-502.

10. Astbury NM, Stevenson EJ, Morris P, et al. (2010) Dose-response effect of a whey protein preload on within-day energy intake in lean subjects. Br J Nutr 104, 1858-1867.

11. Holt S (1999) The effects of high-carbohydrate vs high-fat breakfasts on feelings of fullness and alertness, and subsequent food intake. Int J Food Sci 50, 13-28. 
12. Cecil J, Francis J \& Read N (1999) Comparison of the effects of a high-fat and high-carbohydrate soup delivered orally and intragastrically on gastric emptying, appetite, and eating behaviour. Physiol Behav 67, 299-306.

13. Gibbons C, Caudwell P, Finlayson G, et al. (2013) Comparison of postprandial profiles of ghrelin, active GLP-1, and total PYY to meals varying in fat and carbohydrate and their association with hunger and the phases of satiety. J Clin Endocrinol Metab 98, E847-E855.

14. Essah PA, Levy JR, Sistrun SN, et al. (2007) Effect of macronutrient composition on postprandial peptide YY levels.J Clin Endocrinol Metab 92, 4052-4055.

15. Bowen J, Noakes M, Trenerry C, et al. (2006) Energy intake, ghrelin, and cholecystokinin after different carbohydrate and protein preloads in overweight men. J Clin Endocrinol Metab 91, 1477-1483.

16. Berthoud HR (2006) Homeostatic and non-homeostatic pathways involved in the control of food intake and energy balance. Obesity 14, Suppl. 8, 197S-200S.

17. Finlayson G, King N \& Blundell J (2007) Liking vs. wanting food: importance for human appetite control and weight regulation. Neurosci Biobehav Rev 31, 987-1002.

18. Nijs IM, Muris P, Euser AS, et al. (2010) Differences in attention to food and food intake between overweight/obese and normal-weight females under conditions of hunger and satiety. Appetite 54, 243-254.

19. Davis CA, Levitan RD, Reid C, et al. (2009) Dopamine for 'wanting' and opioids for 'liking': a comparison of obese adults with and without binge eating. Obesity 17, $1220-1225$.

20. Griffioen-Roose S, Mars M, Finlayson G, et al. (2011) The effect of within-meal protein content and taste on subsequent food choice and satiety. Br J Nutr 106, 779-788.

21. Lemmens SG, Martens EA, Born JM, et al. (2011) Lack of effect of high-protein vs. high-carbohydrate meal intake on stress-related mood and eating behavior. Nutr $J \mathbf{1 0}$, 2215-2216.

22. Griffioen-Roose S, Mars M, Siebelink E, et al. (2012) Protein status elicits compensatory changes in food intake and food preferences. Am J Clin Nutr 95, 32-38.

23. Griffioen-Roose S, Smeets PA, van den Heuvel E, et al. (2014) Human protein status modulates brain reward responses to food cues. Am J Clin Nutr 100, 113-122.

24. Green S, Delargy H, Joanes D, et al. (1997) A satiety quotient: a formulation to assess the satiating effect of food. Appetite 29, 291-304.

25. Finlayson G, King N \& Blundell J (2008) The role of implicit wanting in relation to explicit liking and wanting for food: implications for appetite control. Appetite 50, 120-127.

26. Caudwell P, Finlayson G, Gibbons C, et al. (2013) Resting metabolic rate is associated with hunger, self-determined meal size, and daily energy intake and may represent a marker for appetite. Am J Clin Nutr 97, 7-14.

27. Gibbons C, Caudwell P, Finlayson G, et al. (2011) Validation of a new hand-held electronic data capture method for continuous monitoring of subjective appetite sensations. Int J Behav Nutr Phys Act 8, 57-64.

28. Flint A, Raben A, Blundell J, et al. (2000) Reproducibility, power and validity of visual analogue scales in assessment of appetite sensations in single test meal studies. Int J Obes $\mathbf{2 4}$, $38-48$.

29. de Graaf C, Schreurs A \& Blauw YH (1993) Short-term effects of different amounts of sweet and nonsweet carbohydrates on satiety and energy intake. Physiol Behav 54, 833-843.

30. Stubbs J, Rowley E, Hughes D, et al. (1997) Evaluating a new electronic appetite rating system (EARS). Int J Obes 21, 405-415.
31. Johnstone A, Stubbs R \& Harbron C (1996) Effect of overfeeding macronutrients on day-to-day food intake in man. Eur J Clin Nutr 50, 418.

32. Drapeau V, King N, Hetherington M, et al. (2007) Appetite sensations and satiety quotient: predictors of energy intake and weight loss. Appetite 48, 159-166.

33. Drapeau V, Blundell J, Therrien F, et al. (2005) Appetite sensations as a marker of overall intake. Br J Nutr $\mathbf{9 3}$, 273-280.

34. Finlayson G, Arlotti A, Dalton M, et al. (2011) Implicit wanting and explicit liking are markers for trait binge eating. A susceptible phenotype for overeating. Appetite 57, 722-728.

35. Griffioen-Roose S, Finlayson G, Mars M, et al. (2010) Measuring food reward and the transfer effect of sensory specific satiety. Appetite 55, 648-655.

36. Verschoor E, Finlayson G, Blundell J, et al. (2010) Effects of an acute $\alpha$-lactalbumin manipulation on mood and food hedonics in high-and low-trait anxiety individuals. Br J Nutr 104, 595-602.

37. Finlayson G, King N \& Blundell J (2007) Is it possible to dissociate liking'and wanting'for foods in humans? A novel experimental procedure. Physiol Behav 90, 36-42.

38. French SA, Mitchell NR, Finlayson G, et al. (2013) Questionnaire and laboratory measures of eating behavior. Associations with energy intake and BMI in a community sample of working adults. Appetite 72, 50-58.

39. Dalton M, Blundell J \& Finlayson GS (2013) Examination of food reward and energy intake under laboratory and freeliving conditions in a trait binge eating subtype of obesity. Front Psychol 4, 757.

40. Faul F, Erdfelder E, Lang A-G, et al. (2007) G* Power 3: a flexible statistical power analysis program for the social, behavioral, and biomedical sciences. Behav Res Methods 39, 175-191.

41. Raben A, Agerholm-Larsen L, Flint A, et al. (2003) Meals with similar energy densities but rich in protein, fat, carbohydrate, or alcohol have different effects on energy expenditure and substrate metabolism but not on appetite and energy intake. Am J Clin Nutr 77, 91-100.

42. Nutrition TSACo (2015) The Scientific Advisory Committee on Nutrition recommendations on carbohydrates, including sugars and fibre. https://www.gov.uk/government/publications/sacn-carbohydrates-and-health-report

43. Morton G, Cummings D, Baskin D, et al. (2006) Central nervous system control of food intake and body weight. Nature 443, 289-295.

44. Havel PJ, Townsend R, Chaump L, et al. (1999) High-fat meals reduce 24-h circulating leptin concentrations in women. Diabetes 48, 334-341.

45. Romon M, Lebel P, Velly C, et al. (1999) Leptin response to carbohydrate or fat meal and association with subsequent satiety and energy intake. Am I Physiol Endocrinol Metab 277, E855-E861.

46. Romon M, Lebel P, Fruchart J-C, et al. (2003) Postprandial leptin response to carbohydrate and fat meals in obese women. J Am Coll Nutr 22, 247-251.

47. Stunkard A \& Messick S (1985) The three-factor eating questionnaire to measure dietary restraint, disinhibition and hunger. J Psychosom Res 29, 71-83.

48. Martin CK, Rosenbaum D, Han H, et al. (2011) Change in food cravings, food preferences, and appetite during a low carbohydrate and low fat diet. Obesity 19, 1963-1970.

49. McNeil J \& Doucet É (2012) Possible factors for altered energy balance across the menstrual cycle: a closer look at the severity of PMS, reward driven behaviors and leptin variations. Eur J Obstet Gynecol Reprod Biol 163, 5-10. 
50. McNeil J, Cameron JD, Finlayson G, et al. (2013) Greater overall olfactory performance, explicit wanting for high fat foods and lipid intake during the mid-luteal phase of the menstrual cycle. Physiol Behav 112-113, 84-89.

51. Pagoto SL \& Appelhans BM (2013) A call for an end to the diet debates. JAMA 310, 687-688.
52. Lissner L, Levitsky DA, Strupp BJ, et al. (1987) Dietary fat and the regulation of energy intake in human subjects. Am J Clin Nutr 46, 886-892.

53. Tobias DK, Chen M, Manson JE, et al. (2015) Effect of low-fat diet interventions versus other diet interventions on long-term weight change in adults: a systematic review and metaanalysis. Lancet Diabetes Endocrinol 3, 968-979. 